POS $\quad \begin{aligned} & \text { PROCEEDINGS } \\ & \text { OF SCIENCE }\end{aligned}$

\title{
Aerosol in spring-summer-autumn-winter cycles by observation at Yakutsk EAS Array in 2004-2013
}

\section{Stanislav Knurenko}

Yu. G. Shafer Institute of Cosmophysical Research and Aeronomy.

E-mail: knurenkodikfia.sbras.ru

\section{Igor Petrov*}

Yu. G. Shafer Institute of Cosmophysical Research and Aeronomy.

E-mail: igor.petrov@ikfia.sbras.ru

Long-term set of data of aerosol composition and transparency of the atmosphere in the region of Yakutsk analyzed. Season variation of the characteristics are found in the annual cycle. Season variation taken into account in air shower analysis.

The 34th International Cosmic Ray Conference,

30 July- 6 August, 2015

The Hague, The Netherlands

\footnotetext{
* Speaker.
} 


\section{Introduction}

In regions with extreme continental climate, the distribution of aerosol in the air depends on seasonal movements of the wind masses, which are related to the geographical location of the region and to the geophysical situation in the region.

In case of Yakutia with its extreme continental climate and the nature of the anomalous magnetic field latter may have a significant role in shaping the structure of the aerosol, especially during winter. Because of that, the physical model of the atmosphere at a latitude of Yakutsk, is significantly different from the standard, which describes the atmosphere in temperate latitudes [1]. When conducting observations in the optical wavelength range at the Yakutsk one has to take into account all these factors, measuring the aerosol co of the atmosphere and transparency [2,3].

\section{Observation results}

\subsection{Model of the atmosphere in Yakutsk region}

Because of the extremely average temperatures $\left(T=-30-45^{\circ}\right)$ during winter at a latitude of Yakutsk there is a change in height and density of the air temperature profile. The density of the air increases and the temperature inversion occurs at low altitudes ranging from altitudes of $2 \div 4$ $\mathrm{km}$. The quantitative difference in the distribution of air density at altitude we can see in Fig. 2 and Table. 1. In this case, the predominant role in the scattering and absorption of light have near ground layer of the atmosphere. Such a distribution density of air causes a change in slope of the spatial distribution of the Cherenkov light, and for showers, which are below the maximum depth $\mathrm{H}=4,5 \mathrm{~km}\left(\mathrm{X} \sim 550 \mathrm{~g} / \mathrm{cm}^{2}\right)$ to increase the total flux of light compared to standard atmosphere. This feature has to be taken into account when processing the data of air showers[4].

Table 1: Winter atmosphere model in the region of the Yakutsk array.

\begin{tabular}{lllll}
\hline Model of the atmosphere & $\mathrm{T}_{0}$ & $\rho_{0}$ & $\mathrm{n}-1$ & $\mathrm{X}_{0}$ \\
\hline Winter & 243 & $1.453 \cdot 10^{-3}$ & $3.45 \cdot 10^{-4}$ & 1020 \\
Standard & 288 & $1.226 \cdot 10^{-3}$ & $2.92 \cdot 10^{-4}$ & 1030 \\
\hline
\end{tabular}

\subsection{Atmospheric optical thickness analysis during different periods of observation.}

Optical properties of the atmosphere associated with aerial dispersion of optical medium containing particles of different sizes of natural and artificial origin. This is largely affects the propagation of light through the dense layers of the atmosphere where the EAS develops and spreads Cherenkov radiation. Therefore, carrying out regular observations of the atmosphere using a photometer CE 318 and LIDAR at a wavelength of $532 \mathrm{~nm}$ to measure the transparency of the atmosphere in the vicinity of Yakutsk are required [5, 6]. Figure $3 \mathrm{a}$ and $3 \mathrm{~b}$ shows the average values for the month of AOT (atmospheric optical thickness) in the annual cycle of observations for a wavelength of $500 \mathrm{~nm}$. On average, from May to August, the value of AOT equal $\sim 0.20 \pm 0.02$ and decreases to $0.08 \pm 0,02$ in September - November. Long-term observations revealed that the distribution of AOT, reflecting the transition from winter to summer observations, there is a seasonal variation. If the spring and autumn AOT has the lowest value, the value in the winter slightly 


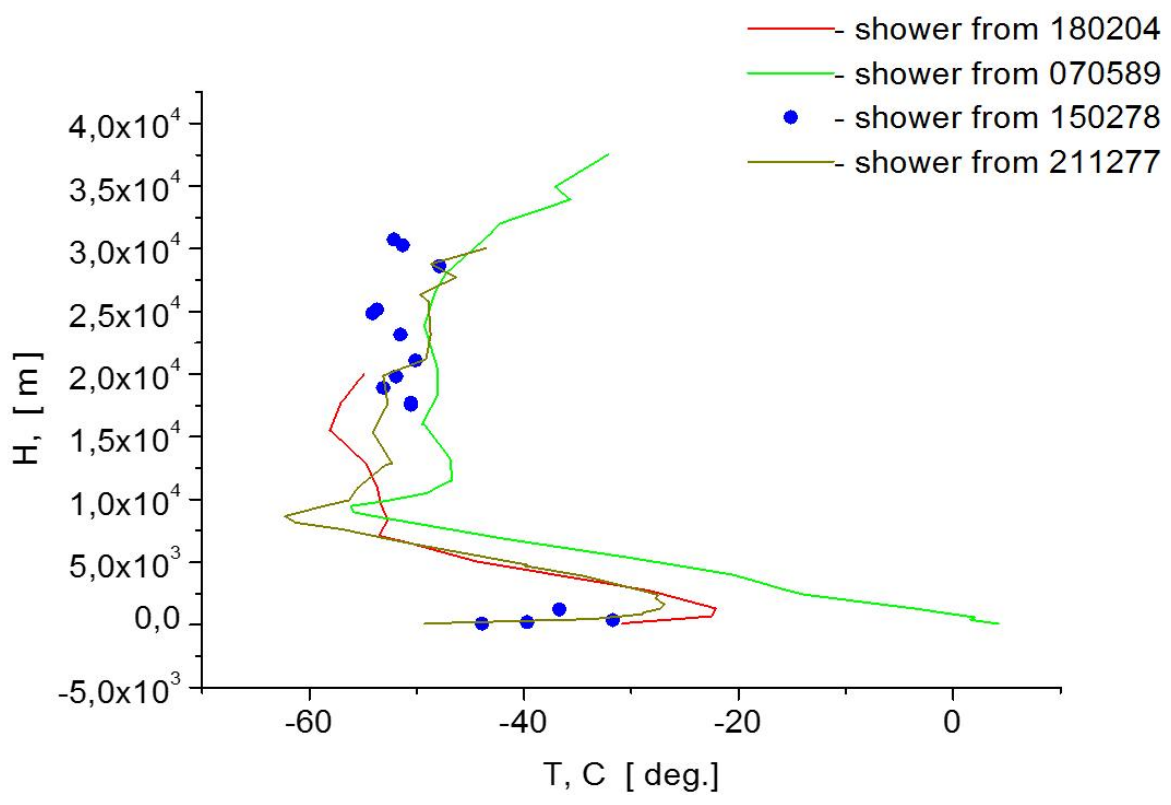

Figure 1: Vertical profile of air temperature distribution at the time of giant air showers registration. Lines are data for December, February and March. There is inversion of the temperature in near ground layer of the atmosphere during winter. Green curve is for spring observations.

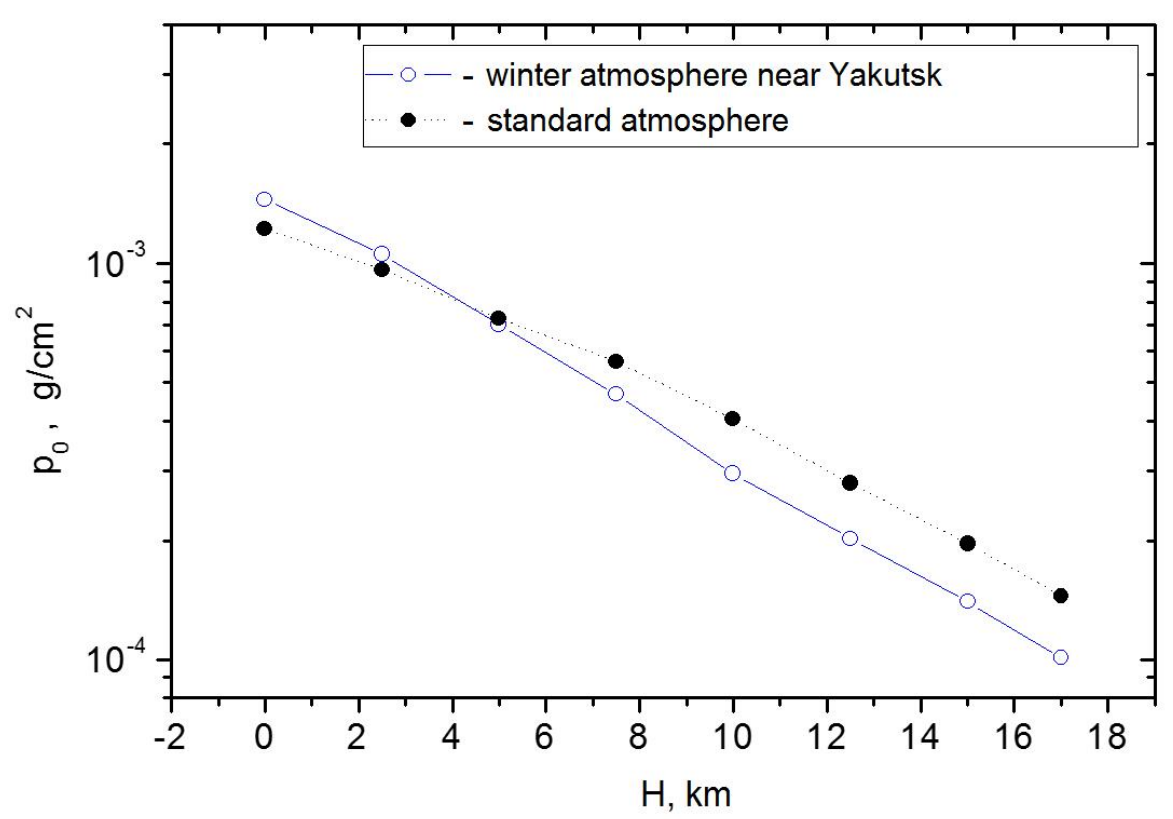

Figure 2: Dependence of air density from height by Yakutsk data in comparison with standard model of the atmosphere. 
increases. This is likely due to changes in the atmosphere, namely the inversion of the temperature and the increase in density of the air near ground level. During this period, the multiple scattering of photons of Cherenkov light increases significantly and this must be taken into account in the registration and processing of signals from the photomultiplier.

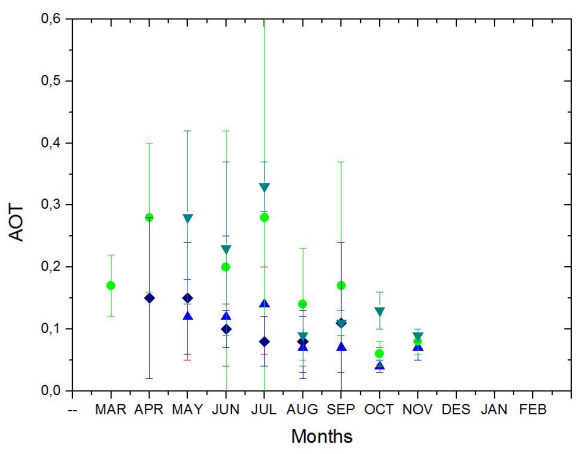

a)

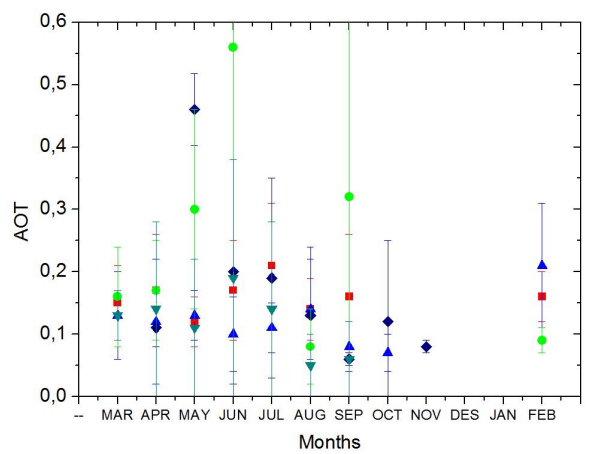

b)

Figure 3: a) The AOT value distribution by years, 2004-2009. Signs represent averaged by month AOT value for $\lambda=500 \mathrm{~nm}$. b) AOT value distribution by years, 2009-2013. Signs represent averaged by month AOT value for $\lambda=500 \mathrm{~nm}$.

\subsection{Transparency of the atmosphere measurement by air shower Cherenkov light of small energies.}

In the paper [7] it was shown that the frequency spectrum of showers with energies $\geq 10^{15}$ $10^{16} \mathrm{eV}$ can be used to control the relative transparency of the atmosphere. For the measurement of absolute transparency at Yakutsk we use a laser at a wavelength of $532 \mathrm{~nm}$ [3].

Analysis of long-term observations of the transparency of the atmosphere in the region of Yakutsk showed seasonal variation of $\tau_{\lambda}$. Fig. 4 shows the transparency of the atmosphere, registered in different years. The data were averaged over a monthly observing sessions. The results show that from year to year coefficient $\tau_{\lambda}$ slightly varies and indicates a stable seasonal variation in atmospheric transparency in the region of Yakutsk. Minimum values $\tau_{\lambda}$ fall on the coldest months - December, January and February, in the remaining months of observations transparency of the atmosphere is considered to be the best [3].

Methods of determining the transparency of the atmosphere by measuring the Cherenkov radiation of extensive air showers in the energy range $10^{15}-10^{16} \mathrm{eV}$ is a priority for the Yakutsk array [8] and is used in the reconstruction of the basic characteristics of EAS at ultrahigh energies $[9,10$, $11]$.

The study of the characteristics of EAS, namely, the longitudinal and lateral development by measuring Cherenkov radiation in the spectral range of $300-800 \mathrm{~nm}$, requires observations of the atmosphere condition. The first is the measurement of AOT and transparency of the atmosphere, because from these characteristics depend on the quality of the Cherenkov light flux measurements, and hence the subsequent measurement of energy and cascade shower development curve $[9,10$, $11]$.

At the Yakutsk the observations of the atmosphere and fixed characteristics such as temperature, pressure, and transparency have been conducting since 1970. A database of continual data 


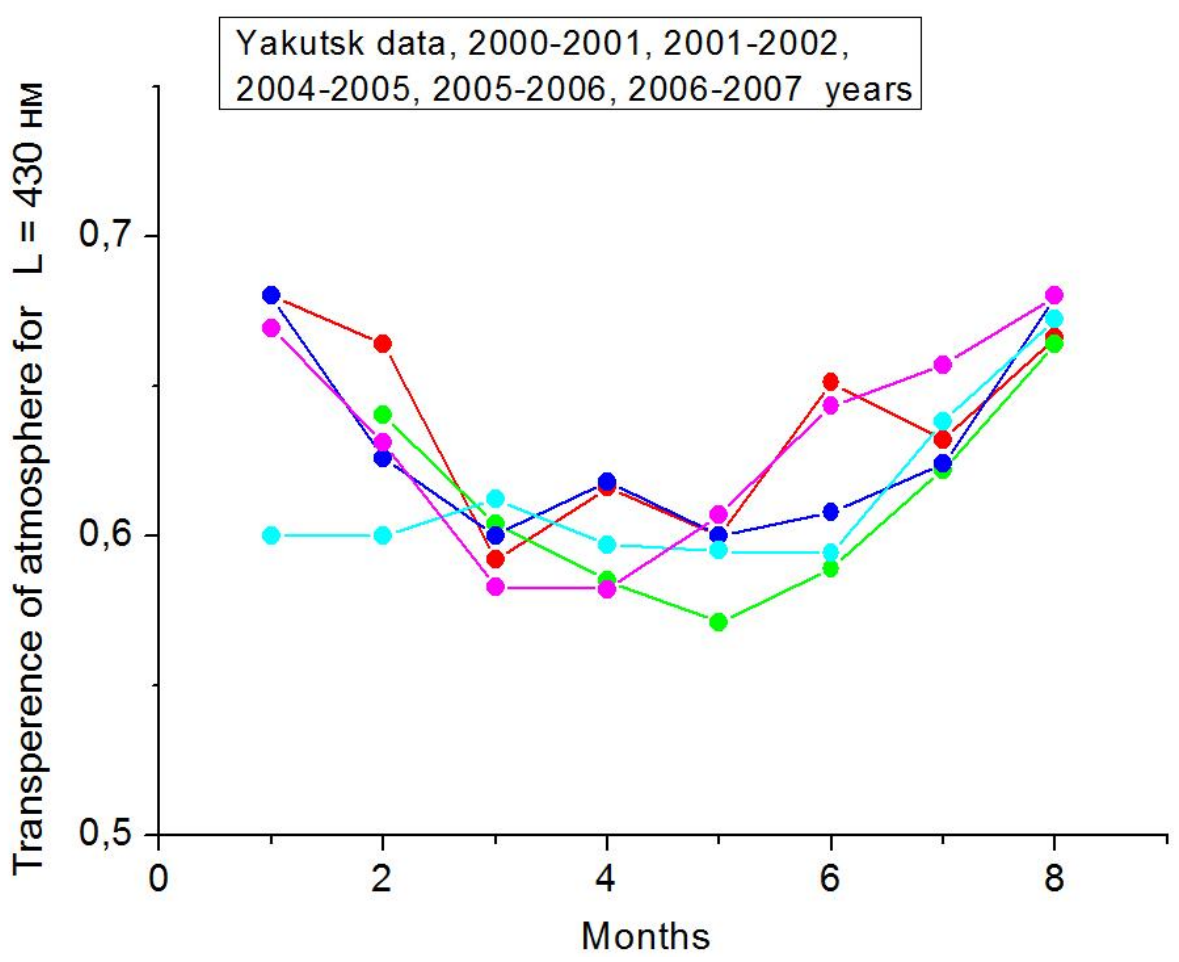

Figure 4: The average monthly atmospheric transmittance for a wavelength of $430 \mathrm{~nm}$. These numbers refer to the month in which the optical measurements are carried out at the Yakutsk array of extensive air showers: 1 - September, October 2.-, 3.- November 4.- December, January 5.-, 6.- February 7.- March 8.- April.

series of these parameters have been created, which allow us to get correction coefficients and apply them in the primary analysis of EAS.

At the same time it is possible to analyze the series of geophysical data and get more information about changing parameters of the atmosphere and climate as a whole [12]. For example, analysis of the AOT and transparency of the atmosphere for a long period of time, revealed a seasonal variation of these parameters in the context of the spring - summer - autumn - winter cycle, which is important for air showers data averaging for the annual and long-term perspective observations as well.

In general, the atmosphere in the region of the Yakutsk array is quite transparent and favorable for measurement of Cherenkov radiation in the optical wavelength range.

\section{Conclusion}

The study of the characteristics of EAS, namely, the longitudinal and lateral development by measuring Cherenkov radiation in the spectral range of $300-800 \mathrm{~nm}$, requires observations of the atmosphere condition. The first is the measurement of AOT and transparency of the atmosphere, because from these characteristics depend on the quality of the Cherenkov light flux measurements, and hence the subsequent measurement of energy and cascade shower development curve $[9,10$, $11]$. 
At the Yakutsk the observations of the atmosphere and fixed characteristics such as temperature, pressure, and transparency have been conducting since 1970. A database of continual data series of these parameters have been created, which allow us to get correction coefficients and apply them in the primary analysis of EAS.

At the same time it is possible to analyze the series of geophysical data and get more information about changing parameters of the atmosphere and climate as a whole [12]. For example, analysis of the AOT and transparency of the atmosphere for a long period of time, revealed a seasonal variation of these parameters in the context of the spring - summer - autumn - winter cycle, which is important for air showers data averaging for the annual and long-term perspective observations as well.

In general, the atmosphere in the region of the Yakutsk array is quite transparent and favorable for measurement of Cherenkov radiation in the optical wavelength range.

\section{References}

[1] V.S. Murzin. Physics of Cosmic Rays. MSU, 285 (1970)

[2] S. M. Sakerin, D. M. Kabanov, M. V. Panchenko et al. Optics of Atmosphere and Ocean. V. 18, 11, 968-975 (2005)

[3] S.P. Knurenko, S.V. Nikolashkin, A.V. Saburov, I.Ye. Sleptsov. Proc. Of SPIE. Vol. 6522 (2006)

[4] S. P. Knurenko, I. S. Petrov. XXI Optics of Atmosphere and Ocean (2015) (in press)

[5] http: // aeronet.gshc.nasa.gov./

[6] S. Knurenko and A. Sabourov 2013 J. Phys.: Conf. Ser. 409012083. doi:10.1088/1742-6596/409/1/012083.

[7] M. N. Dyakonov, S. P. Knurenko, V. A. Kolosov et al. Optics of atmosphere. 4, 8, 868-873 (1991)

[8] F. F. Litsenuk. AS No. 519768, 24 (1976)

[9] S.P. Knurenko, V.A. Kolosov, Z.E. Petrov et al. Proc. 27-th ICRC, Hamburg (Germany), v.1, p.157-160 (2001).

[10] M.N. Dyakonov, S.P. Knurenko, V.A. Kolosov et al. Nuclear Instruments and Meth-s in Phys. Rec. Amst., A248 (1986), v.4, p. 224-226

[11] S. P. Knurenko, A. A. Ivanov, I. Ye. Sleptsov, A. V. Sabourov. JETP Letters. V. 83, 11, 563-567 (2006)

[12] S.P. Knurenko, I.P. Petrov. Proc. SPIE 9292, 20th International Symposium on Atmospheric and Ocean Optics: Atmospheric Physics, 92925B (2014)doi:10.1117/12.2074877 